\title{
CONTINUOUS NILPOTENTS ON TOPOLOGICAL SPACES
}

\author{
R. P. SULLIVAN \\ (Received 27 August 1984; revised 21 October 1986) \\ Communicated by J. H. Rubinstein
}

\begin{abstract}
K. D. Magill has investigated the semigroup generated by the idempotent continuous mappings of a topological space into itself and examined whether this semigroup determines the space to within homeomorphism. By analogy with this (and related work of Bridget Bos Baird) we now consider the semigroup generated by nilpotent continuous partial mappings of a space into itself.
\end{abstract}

1980 Mathematics subject classification (Amer. Math. Soc.): primary 54 C 05; secondary 20 M 20. Keywords and phrases: semigroup, continuous mappings, topological space, isomorphism.

\section{Introduction}

In [2] Howie investigated the semigroup generated by all the idempotents in $\mathscr{T}(X)$, the full transformation semigroup on a set $X$, and subsequently in [4] Magill considered the corresponding subsemigroup of $S(X)$, the semigroup of all continuous self-maps of a topological space $X$.

Certain transformation semigroups contain a "zero" and hence "nilpotents": for example, the semigroup $\mathscr{I}(X)$ of all partial one-to-one transformations of a set $X$ contains the "empty mapping" $\square$ as a zero as well as maps $f$ for which $f^{m}=\square$ for some $m \geqslant 2$, and in [7] we characterised the elements of $\mathscr{I}(X)$ that can be written as a product (under composition) of such "nilpotents". In this paper we commence by investigating the extent to which the semigroup $N_{c}(X)$ generated by all continuous nilpotents whose domains are closed subsets of a topological space $X$ determines the underlying space to within a homeomorphism; we also consider the same question for various subsemigroups of $N_{c}(X)$

(c) 1987 Australian Mathematical Society $0263-6115 / 87 \$ A 2.00+0.00$ 
(this work is analogous to that of Baird [1] and Magill [4]) and remark that the problem of characterising the elements of $N_{c}(X)$ appears insurmountable. However, when we specialise to the closed unit interval $I$ in Section 3 and define a nilpotent to be any continuous map $f: I \rightarrow I$ such that $f^{m}=0$, the zero constant map, we obtain a more complete answer: namely that nilpotents in this sense can be characterised in terms of an order property (using the natural order on $I$ ), and the product of any two nilpotents is again nilpotent.

This paper was completed while visiting Professor J. M. Howie at the University of St. Andrews, Scotland. We thank him and his colleagues for their generous hospitality during the visit.

\section{Partial nilpotents}

If $X$ is a set and $f, g \in \mathscr{P}(X)$, the semigroup (under composition) of all partial transformations of $X$, then $\operatorname{dom}(f g)=\operatorname{dom} g \cap g^{-1}(\operatorname{dom} f)$. This simple fact will enable us to define various semigroups $S$ of continuous partial maps by demanding that the domain of each element of $S$ possess some topological property. We say $f \in \mathscr{P}(X)$ is nilpotent if $f^{m}=\square$ for some $m \geqslant 1$ and that $f$ has index $m$ if $f^{m}=\square$ and $f^{m-1} \neq \square$. Clearly, if $f \in \mathscr{P}(X)$ is nilpotent in this sense, then $\operatorname{dom} f$ and $\operatorname{ran} f$ are proper subsets of $X$.

Our first aim is to show that if $N_{c}(X)$ denotes the semigroup generated (under composition) by all continuous nilpotents whose domains are closed subsets of a topological space $X$, then $N_{c}(X)$ determines all regular $T_{1}$ spaces to within homeomorphism (recall that a space $X$ is regular if for each closed $Q \subseteq X$ and $a \notin Q$, there exist disjoint open $U, V \subseteq X$ such that $Q \subseteq U$ and $a \in V$; and $X$ is $T_{1}$ if and only if the points of $X$ are closed).

We shall need the following result: it is analogous to Lemma 2.5 in [4].

Lemma 1. If $X$ is a regular $T_{1}$ space, then $\left\{f^{-1}(x): x \in X, f \in N_{c}(X)\right\}$ is a basis for the closed subsets of $X$.

Proof. Suppose $Q$ is a non-empty closed subset of $X$, and let $a \notin Q$. Since $X$ is regular, there exist disjoint open subsets $U, V$ of $X$ such that $Q \subseteq U$ and $a \in V$. Define a partial map $f_{a}$ by putting $\operatorname{dom} f_{a}=X \backslash V$ (a closed subset of $X$ containing $U$ ) and $f_{a}(x)=a$ for all $x \notin V$. Then $f_{a}$ is a continuous nilpotent of index 2 , and it is easy to check that $Q=\bigcap\left\{f_{a}^{-1}(a): a \in X \backslash Q\right\}$. 
We now recall a result from [6]: if $X$ is an arbitrary set, any subsemigroup $S$ of $\mathscr{P}(X)$ is briefly referred to as a transformation semigroup and $S$ is said to cover $X$ if for all $x \in X$, there exists an idempotent constant in $S$ with range equal to $\{x\}$.

THEOREM 1. If $S$ is a transformation semigroup covering an arbitrary set $X$, and if $\phi$ is an automorphism of $S$, then there exists a permutation $g: X \rightarrow X$ such that $\phi(f)=g f g^{-1}$ for all $f \in S$.

The following theorem can be proven in a manner identical with that of Theorem 1 in [6], so we omit the details.

THEOREM 2. If $S, T$ are transformation semigroups covering arbitrary sets $X, Y$, respectively, and if $\phi: S \rightarrow T$ is an isomorphism, then there exists a unique bijection $h: X \rightarrow Y$ such that $\phi(f)=h f h^{-1}$ for all $f \in S$. Moreover, if $\phi: S \rightarrow T$ is an isomorphism, and if $\iota_{A} \in S$ for some $A \subseteq X$, then $\phi\left(\iota_{A}\right)=\iota_{h(A)}$, where $h: X \rightarrow Y$ is the bijection associated with $\phi$.

As in [6] we let $A_{x}$ denote the constant map with domain $A$ and range $\{x\}$; moreover, if $A=\{a\}$, we abbreviate $A_{x}$ to $a_{x}$. With this notation, we now observe that if $X$ is a $T_{1}$ space, then $N_{c}(X)$ contains all the maps $x_{x}$ for $x \in X$ (since $x_{x}=y_{x} \circ x_{y}$ for $y \neq x$ ), and so $N_{c}(X)$ covers $X$. Thus we may conclude from Theorem 2 that if $X, Y$ are $T_{1}$ spaces and $\phi: N_{c}(X) \rightarrow N_{c}(Y)$ is an isomorphism, then there exists a bijection $h: X \rightarrow Y$ such that $\phi(f)=h f h^{-1}$ for all $f \in N_{c}(X)$. This establishes part (i) of the next result. The proof of parts (ii) and (iii) is akin to that of Lemma 2.8 [4], so we omit the details (in fact the only difference lies in the use of the injective constants $x_{x}$ rather than the total constants $X_{x}$ ).

LEMMA 2. If $X, Y$ are $T_{1}$ spaces and $\phi$ an isomorphism from $N_{c}(X)$ onto $N_{c}(Y)$, then there exists a bijection $h: X \rightarrow Y$ such that

(i) $\phi(f)=h f h^{-1}$ for each $f \in N_{c}(X)$,

(ii) $h\left(f^{-1}(x)\right)=\phi(f)^{-1}(h(x))$ for each $f \in N_{c}(X)$ and $x \in X$, and

(iii) $h^{-1}\left(g^{-1}(y)\right)=\left(\phi^{-1}(g)\right)^{-1}\left(h^{-1}(y)\right)$ for each $g \in N_{c}(Y)$ and $y \in Y$.

From Lemmas 1 and 2, we readily deduce

THEOREM 3. If $X, Y$ are regular $T_{1}$ spaces, then $\phi: N_{c}(X) \rightarrow N_{c}(Y)$ is an isomorphism if and only if there exists a homeomorphism $h: X \rightarrow Y$ such that $\phi(f)=h f h^{-1}$ for all $f \in N_{c}(X)$. 
We now consider the subsemigroup $N_{c o}(X)$ of $N_{c}(X)$ generated by all continuous maps whose domains are closed-and-open (clopen) subsets of a topological space $X$, and ask whether $N_{c o}(X)$ determines $X$ to within a homeomorphism. In fact, it is easy to show that if $X$ is 0 -dimensional (that is, has a basis consisting of clopen subsets of $X$ ) and also $T_{1}$, then $\left\{f^{-1}(x): x \in X, f \in N_{c o}(X)\right\}$ is a basis for the closed subsets of $X$ (compare Lemma 2.5 [4] and Lemma 1 above). Moreover, if $a \in X$ and $b, c \in X \backslash a$ (an open subset of $X$ ), then there exist clopen subsets $H_{1}$ and $H_{2}$ of $X \backslash a$ such that $b \in H_{1}$ and $c \in H_{2}$. Thus, $E=H_{1} \cup H_{2}$ is a proper clopen subset of $X$ containing $b$ and $c$, and if $d \in X \backslash E$, then $E_{b}=(X \backslash E)_{b} \circ E_{d}$, a product of nilpotents each with index 2 . In other words, if $X$ is a 0-dimensional $T_{1}$ space, then, for each $x, y \in X, N_{c o}(X)$ contains an idempotent constant whose domain contains $\{x, y\}$, and whose range equals $\{x\}$.

The last remark guarantees that if $X, Y$ are 0 -dimensional $T_{1}$ spaces, then $N_{c o}(X)$ and $N_{c o}(Y)$ cover $X$ and $Y$, respectively, and so, by Theorem 2, if $\phi$ : $N_{c o}(X) \rightarrow N_{c o}(Y)$ is an isomorphism, then there exists a bijection $h: X \rightarrow Y$ such that $\phi(f)=h f h^{-1}$ for all $f \in N_{c o}(X)$. To prove that in this situation we have

$$
h\left(f^{-1}(x)\right)=\phi(f)^{-1}(h(x))
$$

for all $x \in X$, we use the discussion of the last paragraph in showing the equivalence of the following statements (compare with [4], page 239):

$$
\begin{aligned}
& y \in h\left(f^{-1}(x)\right) . \\
& y=h(z) \text { and } f(z)=x . \\
& y=h(z) \text { and } f \circ A_{z}=A_{x} \text { for some } A \text { containing }\{x, z\} . \\
& y=h(z) \text { and } \phi(f) \circ \phi\left(A_{z}\right)=\phi\left(A_{x}\right) . \\
& y=h(z) \text { and } \phi(f) \circ B_{h(z)}=B_{h(x)} \text { for some } B \text { containing }\{h(x), h(z)\} . \\
& y=h(z) \text { and } \phi(f)(h(z))=h(x) . \\
& y \in \phi(f)^{-1}(h(x)) .
\end{aligned}
$$

That is, when $X, Y$ are 0 -dimensional $T_{1}$ spaces, we can establish a result for isomorphisms between $N_{c o}(X)$ and $N_{c o}(Y)$ that is entirely similar to Lemma 2 above. We put all this together in

THeOREM 4. If $X, Y$ are 0-dimensional $T_{1}$ spaces, then $\phi: N_{c o}(X) \rightarrow N_{c o}(Y)$ is an isomorphism if and only if there exists a homeomorphism $h: X \rightarrow Y$ such that $\phi(f)=h f h^{-1}$ for all $f \in N_{c o}(X)$.

Our next aim is to provide conditions under which $N I_{F}(X)$, the semigroup generated by all nilpotent homeomorphisms whose domains are closed subsets of a topological space $X$, determines $X$ to within a homeomorphism of $X$. However, we first note that in Theorem 2 , when we restrict our attention to inverse 
subsemigroups of $\mathscr{I}(X)$, the semigroup of all injective partial transformations of an arbitrary set $X$, we obtain Theorem 3.1 of [1]. Since a particular example of an inverse semigroup covering $X$ is the semigroup $I_{F}(X)$ of all homeomorphisms whose domains are closed subsets of a $T_{1}$ space $X$, we obtain the next result directly from Theorem 2 (compare with Corollary 3.5 in [1]).

Corollary 1. If $X, Y$ are $T_{1}$ spaces, then $\phi: I_{F}(X) \rightarrow I_{F}(Y)$ is an isomorphism if and only if there exists a homeomorphism $h: X \rightarrow Y$ such that $\phi(f)=h_{f h}^{-1}$ for all $f \in I_{F}(X)$.

Since $N I_{F}(X)$ is also an inverse semigroup and since, when $X$ is $T_{1}, N I_{F}(X)$ covers $X$, we readily obtain (using results analogous to Lemmas 1 and 2 to show that $h$ is a homeomorphism)

CoRollaRY 2. If $X$, Y are regular $T_{1}$ spaces, then $\phi: N I_{F}(X) \rightarrow N I_{F}(Y)$ is an isomorphism if and only if there exists a homeomorphism $h: X \rightarrow Y$ such that $\phi(f)=h f h^{-1}$ for all $f \in N I_{F}(X)$.

We now prove a result similar to Corollary 2.9 in [4].

THEOREM 5. If $X, Y$ are regular $T_{1}$ spaces, then any isomorphism from $N I_{F}(X)$ onto $N I_{F}(Y)$ has a unique extension to an isomorphism from $I_{F}(X)$ onto $I_{F}(Y)$.

Proof. Suppose $\phi$ is an isomorphism from $N I_{F}(X)$ onto $N I_{F}(Y)$. By Corollary 2 there is a homeomorphism $h: X \rightarrow Y$ such that $\phi(f)=h f h^{-1}$ for every $f \in N I_{F}(X)$. We extend $\phi$ to an isomorphism $\bar{\phi}: I_{F}(X) \rightarrow I_{F}(Y)$ by defining $\bar{\phi}(f)=h h^{-1}$ for each $f \in I_{F}(X)$; the extension is unique since any isomorphism $\psi$ from $I_{F}(X)$ onto $I_{F}(Y)$ that agrees with $\phi$ on $N I_{F}(X)$ must also agree with $\phi$ on $\left\{x_{x}: x \in X\right\}$, and this suffices to prove that $\psi=\bar{\phi}$.

REMARK 1. As above, it is possible to show that the semigroup generated by all nilpotent homeomorphisms whose domains are clopen subsets of a 0 -dimensional $T_{1}$ space determines that space to within a homeomorphism.

REMARK 2. In general, if $X$ is an arbitrary set and $f \in \mathscr{P}(X)$ is nilpotent, then $h f h^{-1} \in \mathscr{P}(X)$ is nilpotent for every permutation $h: X \rightarrow Y$. With this in mind, it readily follows that if $X$ is a regular $T_{1}$ space, then the automorphism group of $N_{c}(X)$ is isomorphic to the group of all homeomorphisms from $X$ into $X$ (compare with Corollary 2.10 [4]). Similar statements could also be made about the automorphism groups of $N I_{F}(X)$ and $N_{c o}(X)$ for suitable spaces $X$. 
In [7] we characterised products of nilpotents in $\mathscr{I}(X)$ as those $f \in \mathscr{I}(X)$ such that $|X \backslash \operatorname{ran} f| \geqslant \operatorname{rank} f$ and $|X \backslash \operatorname{dom} f| \geqslant \operatorname{rank} f$, where $\operatorname{rank} f$ is defined to be $|\operatorname{ran} f|$. Unfortunately, this latter condition does not suffice to ensure that if $f \in I_{F}(X)$, and $f$ satisfies the condition, then $f \in N I_{F}(X)$. One problem entails deciding when, for $f \in I_{F}(X)$, there exists a closed set $Q \subseteq X \backslash \operatorname{dom} f$ and a (nilpotent) homeomorphism $g$ : $\operatorname{dom} f \rightarrow Q$ (compare the proof of Theorem 3 in [7]). For example, suppose that $X=A \cup B$ is a disjoint union of two sets with cardinal equal to $|X| \geqslant \boldsymbol{\aleph}_{0}$. If we take $X, A$ and $\square$ as the open subsets of $X$, then $N I_{F}(X)=\{\square\}$ since $I_{F}(X)=\left\{\iota_{X}, \iota_{B}, \square\right\}$, and, although $\iota_{B}$ satisfies the abovementioned condition, it cannot be written as a product of non-zero nilpotents in $I_{F}(X)$ (since there are none!). For a more interesting example, let $X$ be an infinte set and fix a proper subset $Y$ of $X$ for which $|Y|=|X|$. We topologise $X$ with the smallest $\left(T_{1}\right)$ topology under which $Y$ and all finite subsets of $X$ are closed. In this event, $Q \subseteq X$ is closed if and only if $Q$ is finite or $Q=Y \cup F$ for some finite $F \subseteq X$. Suppose $\iota_{Y}=f_{1} \cdots f_{n}$, a product of nilpotents in $I_{F}(X)$. Then $Y \subseteq$ $\operatorname{dom} f_{n}$, and since $f_{n} \in I_{F}(X)$, we deduce that dom $f_{n}=Y \cup E$ and $\operatorname{ran} f_{n}=Y \cup$ $F$ for some finite sets $E, F \subseteq X$. However, since $f_{n}$ can be regarded as a nilpotent element of $\mathscr{I}(Z)$, where $Z=Y \cup E \cup F$, we must have $\left|Z \backslash \operatorname{dom} f_{n}\right|=|F \backslash E|$ $\geqslant \operatorname{rank} f_{n}=|X|$, which is plainly a contradiction.

Of course if we sufficiently narrow our vision, then everything will work. Let us say that a space $X$ is bonded if for every proper closed $A \subseteq X$, there exists some closed $B \subseteq X \backslash A$ such that $|B|=|A|$. Rather surprisingly, such spaces exist in abundance. For example, suppose $X, Y$ are sets such that $|X|>|Y| \geqslant \aleph_{0}$, and let us topologise $X$ by saying that $A \subseteq X$ is closed if and only if $|A| \leqslant|Y|$. Clearly, $X$ with this topology is a bonded space, and for such $X$ we shall write $X=X_{k}$ where $k=|Y|$ and call $X$ a bonded $k$-space. (We thank Dr. Peter Jupp for giving us this example.) However, not every bonded space is an $X_{k}$ for some cardinal $k$ : for instance, any countably infinite set $X$, topologised by saying that $A \subseteq X$ is closed if and only if $A$ is finite, is a bonded space and does not equal any $X_{k}$ by definition.

Every bonded $k$-space is $T_{1}$ (obviously) and normal: if $A, B \subseteq X_{k}$ are disjoint closed sets then $|X \backslash(A \cup B)|=|X|$, and we can write $X \backslash(A \cup B)=P \cup \dot{\cup} Q$, where $|P|=|Q|=|X|$. In this event, $P \cup A$ and $Q \cup B$ are both open in $X_{k}$ and contain $A$ and $B$, respectively. However, not every normal space is a bonded $k$-space: any set $X$ with the discrete topology is normal and cannot be an $X_{k}$ for any cardinal $k<|X|$.

Bonded $k$-spaces also have the property: if $A, B \subseteq X_{k}$ are closed with the same cardinal, and $f: A \rightarrow B$ is any bijection, then $f$ is a homeomorphism. For, if $C \subseteq X_{k}$ is closed, then $f(A \cap C)=B \cap f(C)$ and, by virtue of the cardinality condition, both $f(C)$ and $B \cap f(C)$ are closed in $X_{k}$ : that is, $f$ is a closed map. Likewise, if $D \subseteq X_{k}$ is closed, then $f^{-1}(B \cap D)=A \cap f^{-1}(D)$, where $f^{-1}(D)$ is 
closed. (Note that this is true because $f$ is injective; thus, $f$ is a continuous map.) Finally, observe that since both $|\operatorname{ran} f|$ and $|\operatorname{dom} f|$ are less than $k$ and $k<|X|$, we have $|X \backslash \operatorname{ran} f|=|X \backslash \operatorname{dom} f|=|X|$ : that is, by [7] Theorem 3, $f$ is a product of nilpotents in $I_{F}\left(X_{k}\right)$; in fact, since $(X \backslash \operatorname{ran} f) \cap(X \backslash \operatorname{dom} f)$ has cardinal equal to $|X|$, it is easy to see that $f$ is actually a product of just two nilpotents each with index 2 .

\section{Total nilpotents}

As noted by Kuratowski [3, pp. 285-286], "every 0-dimensional space is homeomorphic to a subspace of the Cantor discontinuum", and "every topological space with cardinal less than c (the continuum) is 0-dimensional". Since therefore we hae to some extent been working in Section 2 with spaces of cardinal less than $\mathbf{c}$, it seems natural now to focus the light on spaces with cardinal $\mathbf{c}$. In particular, we here study nilpotents defined on $I=[0,1]$, the closed unit interval. For this context it seems inappropriate to consider "partial" nilpotents. Instead, we let $S_{0}(I)$ denote the semigroup (under composition) of all continuous self-maps of $I$ that fix 0 , and observe that $S_{0}(I)$ contains as "zero" the mapping 0 : $[0,1] \rightarrow 0$. In this section we characterise the "nilpotents" of $S_{0}(I)$ and show that the product of any two nilpotents is again nilpotent.

An $f \in S_{0}(I)$ will be called nilpotent if $f^{m}=f \circ \cdots \circ f=0$ for some $m \geqslant 1$; we say $f$ has index $m$ if $f^{m}=0$ but $f^{m-1} \neq 0$, and we let $N(I)$ denote the set of all nilpotents in $S_{0}(I)$.

Clearly nilpotents in this sense are never surjective; to say more about them, we shall use (without mention) the fact that real-valued continuous functions map closed intervals to (possibly degenerate) closed intervals and possess the Intermediate Value Property.

LEMMA 3. If $f \in N(I)$ and $f \neq 0$, then there exist $a>0$ and $b<1$ such that $f([0, a])=0$ and $f(I)=[0, b]$. Moreover, if $a_{f}=\sup \{a \in I: f([0, a])=0\}$, then $f(x)<x$ for all $x>a_{f}$.

Proof. Suppose $f^{m+1}=0$ and $f^{m} \neq 0$. Then $f^{m}:[0,1] \rightarrow[u, v]$ for some $u, v \in I$ and, since $f^{m}(f(0))=0$, we deduce that $0 \in[u, v]$. Hence $u \neq v$, and in fact, $u=0$; since $f^{m+1}=0$, we obtain $f([0, v])=0$ for $v>0$, and consequently $f:[0,1] \rightarrow[0, b]$ for some $b<1$.

Now, the set $\{a \in I: f([0, a])=0\}$ is non-empty and bounded above by 1 ; hence, $a_{f}$ exists and it equals 1 if and only if $f=0$. If $x>a_{f}>0$, then $f([0, x])=\left[0, c_{1}\right]$ for some $c_{1} \in I$. If $x \geqslant c_{1}$, then $f(x) \leqslant c_{1} \leqslant x$, and the result 
follows (note that $f(x) \neq x$ since $f$ is nilpotent and $x \neq 0$ ); on the other hand, if $x<c_{1}$, then $f\left(\left[0, c_{1}\right]\right)=\left[0, c_{2}\right]$, and again, if $x \geqslant c_{2}$, the result follows, while if $x<c_{2}$, we repeat the argument. This process must stop, since otherwise we obtain a sequence $\left\{c_{n}\right\}$ for which $0<x<c_{n}$ and $f^{n}([0, x])=\left[0, c_{n}\right]$ for all $n$, contradicting the nilpotency of $f$.

We note for future reference that the above proof actually shows a little more: namely, if $f \in N(I)$ and $x>a_{f}$, then $f([0, x])=[0, c]$, where $c \leqslant x$.

Lemma 4. If $f \in S_{0}(I)$, and if there exists $a>0$ such that $f([0, a])=0$ and $f(x)<x$ for all $x>a$, then $f$ is nilpotent.

Proof. If $a=1$, then $f=0$, so we suppose that $a<1$ and let $f\left(x_{1}\right)$ be a global maximum of $f$ on $[a, 1]$. Then $a<x_{1}$ (otherwise $f=0$, contrary to supposition), and $f\left(x_{1}\right)<x_{1}$. If $f\left(x_{1}\right) \leqslant a$, then $f^{2}=0$ since $f:[0,1] \rightarrow\left[0, x_{1}\right]$. So we suppose that $a<f\left(x_{1}\right)$ and let $f\left(x_{2}\right)$ be a global maximum of $f$ on $\left[a, f\left(x_{1}\right)\right]$. Then $f\left(\left[a, f\left(x_{1}\right)\right]\right)=\left[0, f\left(x_{2}\right)\right]$, and $f\left(x_{2}\right)<x_{2} \leqslant f\left(x_{1}\right)$. If $f\left(x_{2}\right) \leqslant a$, then we obtain $f^{3}=0$. So we suppose $a<f\left(x_{2}\right)$ and repeat the argument. In this fashion we can generate a strictly descending sequence $\left\{x_{n}\right\}$ bounded below by $a$ :

$$
a<\cdots \leqslant f\left(x_{n}\right)<x_{n} \leqslant f\left(x_{n-1}\right)<x_{n-1} \leqslant \cdots<x_{1} .
$$

Suppose $y=\lim x_{n}$. Then $y \geqslant a$ : if $y=a$, we have $a \leqslant \lim f\left(x_{n}\right)=f(y)=0$, contradicting $a>0$; while if $y>a$, we have $y=\lim f\left(x_{n}\right)=f(y)$, contradicting the fact that $f(x)<x$ whenever $x>a$. Hence, the foregoing process must stop in a finite number of steps and when it does we deduce that $f$ is nilpotent.

We now use the characterisation of nilpotents presented in Lemmas 3 and 4 to show that $N(I)$ is a subsemigroup of $S_{0}(I)$.

THEOREM 6. If $f, g \in S_{0}(I)$ are nilpotent, then $f g$ is also.

Proof. If either $f$ or $g$ equals 0 then $f g=0$. So, we suppose that $f \neq 0$ and $g \neq 0$, in which case $0<a_{g}<1$ and $f g\left(\left[0, a_{g}\right]\right)=0$. Let $d=\sup \{z \in I$ : $f g([0, z])=0\}$. Then $0<a_{g} \leqslant d \leqslant 1$, and $d=1$ if and only if $f g=0$. If $d<1$ and $d<x$ then $a_{g}<x$ and $g(x)<x$ by Lemma 3; if $g(x) \leqslant a_{f}$ then $f g(x)=0$, contradicting the choice of $d$. Hence $a_{f}<g(x)$ and we obtain $f g(x)<g(x)<x$, as required.

The automorphisms of various subsemigroups of $S(I)$ have been determined in [5] and [8]. We now provide evidence to suggest that every automorphism $\phi$ of $N(I)$ is "inner": that is, there exists a homeomorphism $k: I \rightarrow I$ such that $\phi(f)=k f k^{-1}$ for all $f \in N(I)$ (compare Remark 2 above). For this purpose we 
let $E(a, b)$ denote the set of all $f \in N(I)$ for which $a_{f}=a$ and $f([0,1])=[0, b]$. The next result provides a useful characterisation of the sets $E(a, a)$ that readily shows they are preserved by automorphisms of $N(I)$.

LEMMA 5. If $f \in N(I)$ then $f \in E(a, a)$ for some $a \in I$ if and only if $f g f=0$ for all $g \in N(I)$.

Proof. Suppose $f \in E(a, a)$ and let $g \in E(b, c)$. If $a \leqslant b$, then $g f=0$ and if $b<a$ then $g([0, a])=[0, d]$ where $d \leqslant a$ and so $f g f=0$. Conversely, suppose $f g f=0$ for all $g \in N(I)$ where $f \in E(a, b)$ and $f \neq 0$. If $a<b$ then $f(t) \neq 0$ for some $t$ where $a<t<b$; we then let $0<s<a$ and define a map $g$ via: $g(x)=0$ for $0 \leqslant x \leqslant a, g(x)=s(x-a) /(t-a)$ for $a \leqslant x \leqslant t, g(x)=t$ for $b \leqslant x \leqslant 1$, and

$$
g(x)=\frac{t(x-t)+s(b-x)}{b-t} \text { for } t \leqslant x \leqslant b .
$$

Then $g^{3}=0$ and if $f(w)=b$ then $f g f(w) \neq 0$; a dual argument for the case $b<a$ concludes the proof.

To prove the next result we shall use the obvious fact that whenever $0<a, b, c$ $<1$, we have: $a \leqslant b$ if and only if $E(b, c) \cdot E(a, a)=0$.

LEMMA 6. Suppose $\phi$ is an automorphism of $N(I)$. Then there is a strictly increasing function $k: I \rightarrow I$ such that $\phi(E(a, b))=E(k(a), k(b))$ for all $a, b$ with $0<a, b<1$.

Proof. For $0<a, b<1$ we define $k(a)=b$ if and only if $\phi(E(a, a))=$ $E(b, b)$. From the above remark, $x=y$ if and only if $E(x, x) \cdot E(y, y)=$ $E(y, y) \cdot E(x, x)=0$ : this and Lemma 5 can be used to show that $k$ is a well-defined permutation of $I \backslash\{0,1\}$. We put $k(0)=0$ and $k(1)=1$, and again use the remark to deduce that $k$ is strictly increasing.

\section{References}

[1] B. B. Baird, 'Isomorphisms between inverse semigroups of injective transformations,' $J$. Austral. Math. Soc. (Series A) 23 (1977), 194-201.

[2] J. M. Howie, 'The subsemigroup generated by the idempotents of a full transformation semigroup', J. London Math. Soc. 41 (1966), 707-716.

[3] K. Kuratowski, Topology, Vol. 1 (Academic Press, New York, 1966).

[4] K. D. Magill, Jr., 'Semigroups of functions generated by idempotents', J. London Math. Soc. 44 (1969), 236-242. 
[5] K. D. Magill, Jr., 'Homomorphisms from $S(X)$ onto $S(Y)$ ', Canad. J. Math. 29 (1977), 615-625.

[6] R. P. Sullivan, 'Automorphisms of transformation semigroups', J. Austral. Math. Soc. (Series A) 20 (1975), 77-84.

[7] R. P. Sullivan, 'Semigroups generated by nilpotent transformations', submitted.

[8] G. R. Wood, 'Automorphisms of semigroups of continuous functions', J. Austral. Math. Soc. (Series A) 29 (1980), 301-309.

\section{Department of Mathematics}

University of Western Australia

Nedlands, 6009

Australia 